\title{
On generalized Srivastava-Owa fractional operators in the unit disk
}

Rabha W Ibrahim

Correspondence:

rabhaibrahim@yahoo.com Institute of Mathematical Sciences, University Malaya, 50603 Kuala Lumpur, Malaysia

\begin{abstract}
This article introduces a generalization for the Srivastava-Owa fractional operators in the unit disk. Conditions are given for the fractional integral operator to be bounded in Bergman space. Some properties for the above operator are also provided. Moreover, applications of these operators are posed in the geometric functions theory and fractional differential equations.
\end{abstract}

\section{Introduction}

Recently, the theory of fractional calculus has found interesting applications in the theory of analytic functions. The classical definitions of fractional operators and their generalizations have fruitfully been applied in obtaining, for example, the characterization properties, coefficient estimates [1], distortion inequalities [2] and convolution structures for various subclasses of analytic functions and the works in the research monographs. In [3], Srivastava and Owa gave definitions for fractional operators (derivative and integral) in the complex z-plane $\mathbf{C}$ as follows:

Definition 1.1. The fractional derivative of order $\alpha$ is defined, for a function $f(z)$, by

$$
D_{z}^{\alpha} f(z):=\frac{1}{\Gamma(1-\alpha)} \frac{d}{d z} \int_{0}^{z} \frac{f(\zeta)}{(z-\zeta)^{\alpha}} d \zeta ; \quad 0 \leq \alpha<1,
$$

where the function $f(z)$ is analytic in simply-connected region of the complex z-plane $\mathrm{C}$ containing the origin, and the multiplicity of $(z-\zeta)^{-\alpha}$ is removed by requiring $\log (z-$

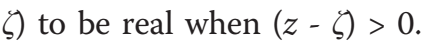

Definition 1.2. The fractional integral of order $\alpha$ is defined, for a function $f(z)$, by

$$
I_{z}^{\alpha} f(z):=\frac{1}{\Gamma(\alpha)} \int_{0}^{z} f(\zeta)(z-\zeta)^{\alpha-1} d \zeta ; \quad \alpha>0,
$$

where the function $f(z)$ is analytic in simply-connected region of the complex z-plane (C) containing the origin, and the multiplicity of $(z-\zeta)^{\alpha-1}$ is removed by requiring log $(z-\zeta)$ to be real when $(z-\zeta)>0$.

Remark 1.1. From Definitions 1.1 and 1.2, we have $D_{z}^{0} f(z)=f(0), \quad \lim _{\alpha \rightarrow 0} I_{z}^{\alpha} f(z)=f(z)$ and $\lim _{\alpha \rightarrow 0} D_{z}^{1-\alpha} f(z)=f^{\prime}(z)$. Moreover,

$$
D_{z}^{\alpha}\left\{z^{\mu}\right\}=\frac{\Gamma(\mu+1)}{\Gamma(\mu-\alpha+1)}\left\{z^{\mu-\alpha}\right\}, \quad \mu>-1 ; \quad 0 \leq \alpha<1
$$

(C) 2011 Ibrahim; licensee Springer. This is an Open Access article distributed under the terms of the Creative Commons Attribution License (http://creativecommons.org/licenses/by/2.0), which permits unrestricted use, distribution, and reproduction in any medium, provided the original work is properly cited. 
and

$$
I_{z}^{\alpha}\left\{z^{\mu}\right\}=\frac{\Gamma(\mu+1)}{\Gamma(\mu-\alpha+1)}\left\{z^{\mu-\alpha}\right\}, \quad \mu>-1 ; \quad 0 \leq \alpha<0 .
$$

Further properties of these operators can be found in $[4,5]$.

\section{Generalized integral operator}

For $0<p<1$, the Bergman space $\mathcal{A}^{p}$ is the set of functions $f$ analytic in the unit disk $U$ $:=\{z: z \in \mathbf{C} ;|z|<1\}$ with $\|f\|_{\mathcal{A}^{p}}^{p}<\infty$, where the norm is defined by

$$
\|f\|_{\mathcal{A}^{p}}^{p}=\frac{1}{\pi} \int_{U}|f(z)|^{p} d A<\infty, \quad z \in U,
$$

and $d A$ is denoted Lebesgue area measure.

To derive a formula for the generalized fractional integral, consider for natural $n \in$ $\mathbf{N}=\{1,2, \ldots\}$ and real $\mu$, the $n$-fold integral of the form

$$
I_{z}^{\alpha, \mu} f(z)=\int_{0}^{z} \zeta_{1}^{\mu} d \zeta_{1} \int_{0}^{\zeta_{1}} \zeta_{2}^{\mu} d \zeta_{2} \ldots \int_{0}^{\zeta_{n-1}} \zeta_{n}^{\mu} f\left(\zeta_{n}\right) d \zeta_{n} .
$$

By employing the Dirichlet technique yields

$$
\int_{0}^{z} \zeta_{1}^{\mu} d \zeta_{1} \int_{0}^{\zeta_{1}} \zeta^{\mu} f(\zeta) d \zeta=\int_{0}^{z} \zeta^{\mu} f(\zeta) d \zeta \int_{\zeta}^{z} \zeta_{1}^{\mu} d \zeta_{1}=\frac{1}{\mu+1} \int_{0}^{z}\left(z^{\mu+1}-\zeta^{\mu+1}\right)^{n-1} \zeta^{\mu} f(\zeta) d \zeta
$$

Repeating the above step $n$ - 1 times we have

$$
\int_{0}^{z} \zeta_{1}^{\mu} d \zeta_{1} \int_{0}^{\zeta_{1}} \zeta_{2}^{\mu} d \zeta_{2} \ldots \int_{0}^{\zeta_{n-1}} \zeta_{n}^{\mu} f\left(\zeta_{n}\right) d \zeta_{n}=\frac{(\mu+1)^{1-n}}{(n-1) !} \int_{0}^{z}\left(z^{\mu+1}-\zeta^{\mu+1}\right)^{n-1} \zeta^{\mu} f(\zeta) d \zeta
$$

which implies the fractional operator type

$$
I_{z}^{\alpha, \mu} f(z)=\frac{(\mu+1)^{1-\alpha}}{\Gamma(\alpha)} \int_{0}^{z}\left(z^{\mu+1}-\zeta^{\mu+1}\right)^{\alpha-1} \zeta^{\mu} f(\zeta) d \zeta,
$$

where $a$ and $\mu \neq-1$ are real numbers and the function $f(z)$ is analytic in simply-connected region of the complex z-plane $\mathbf{C}$ containing the origin, and the multiplicity of $\left(z^{\mu+1}-\zeta^{\mu+1}\right)^{-\alpha}$ is removed by requiring $\log \left(z^{\mu+1}-\zeta^{\mu+1}\right)$ to be real when $\left(z^{\mu+1}-\zeta^{\mu+1}\right)>0$. When $\mu=0$, we arrive at the standard Srivastava-Owa fractional integral, which is used to define the Srivastava-Owa fractional derivatives.

Theorem 2.1. Let $\alpha>0,0<p<\infty$ and $\mu \in \mathbf{R}$. Then, the operator $I_{z}^{\alpha, \mu}$ is bounded in $\mathcal{A}^{p}$ and

$$
\left\|I_{z}^{\alpha, \mu} f(z)\right\|_{\mathcal{A}^{p}}^{p} \leq C\|f(z)\|_{\mathcal{A}^{p}}^{p},
$$

where

$$
C:=\int_{0}^{1}\left|\frac{(\mu+1)^{1-\alpha}}{\Gamma(\alpha)}\left(1-w^{\mu+1}\right)^{\alpha-1} w^{\mu} d w\right|^{p} .
$$


Proof. Assume that $f(z) \in \mathcal{A}^{p}$. Then, we have

$$
\begin{aligned}
\left\|I_{z}^{\alpha, \mu} f(z)\right\|_{\mathcal{A}^{p}}^{p} & =\frac{1}{\pi} \int_{U}\left|I_{z}^{\alpha, \mu} f(z)\right|^{p} d A \\
& =\frac{1}{\pi} \int_{0}^{1}\left|\frac{(\mu+1)^{1-\alpha}}{\Gamma(\alpha)} \int_{0}^{z}\left(z^{\mu+1}-\zeta^{\mu+1}\right)^{\alpha-1} \zeta^{\mu} f(\zeta) d \zeta\right|^{p} d A \\
& =\frac{1}{\pi} \int_{0}^{1}\left|\frac{(\mu+1)^{1-\alpha}}{\Gamma(\alpha)} \int_{0}^{z}\left(1-\frac{\zeta^{\mu+1}}{z^{\mu+1}}\right)^{\alpha-1} z^{(\mu+1)(\alpha-1)} \zeta^{\mu} f(\zeta) d \zeta\right|^{p} d A .
\end{aligned}
$$

Let $w:=\frac{\zeta}{z}$, then we obtain

$$
\begin{aligned}
\left\|I_{z}^{\alpha, \mu} f(z)\right\|_{\mathcal{A}^{p}}^{p} & =\frac{1}{\pi} \int_{0}^{1}\left|\frac{(\mu+1)^{1-\alpha}}{\Gamma(\alpha)} \int_{0}^{\frac{\zeta}{w}}\left(1-w^{\mu+1}\right)^{\alpha-1} z^{(\mu+1)(\alpha)} w^{\mu} f(w z) d w\right|^{p} d A \\
& \leq \frac{1}{\pi} \int_{0}^{1}\left|\frac{(\mu+1)^{1-\alpha}}{\Gamma(\alpha)} \int_{U}\left(1-w^{\mu+1}\right)^{\alpha-1} w^{\mu} f(w z) d w\right|^{p} d A \\
& \leq \int_{0}^{1}\left|\frac{(\mu+1)^{1-\alpha}}{\Gamma(\alpha)} \int_{U}\left(1-w^{\mu+1}\right)^{\alpha-1} w^{\mu} d w\right|^{p}\left(\frac{1}{\pi} \int_{U}|f(\zeta)|^{p} d A\right) \\
& :=C\|f\|_{\mathcal{A}^{p}}^{p}
\end{aligned}
$$

This completes the proof.

Next, we give semigroup properties of the integral operator.

Theorem 2.2. Let $f$ be analytic in the unit disk. Then, operator (2) satisfies

$$
I_{z}^{\alpha+\beta, \mu} f=I_{z}^{\alpha, \mu} I_{z}^{\beta, \mu} f, \quad \alpha>0, \beta>0 .
$$

Proof. For function $f$ by using Dirichlet technique yields

$$
\begin{aligned}
I_{z}^{\alpha, \mu} I_{z}^{\beta, \mu} f(z) & =\frac{(\mu+1)^{(1-\alpha)}}{\Gamma(\alpha)} \int_{0}^{z}\left(z^{\mu+1}-\zeta^{\mu+1}\right)^{\alpha-1} \zeta^{\mu} I_{\zeta}^{\beta, \mu} f(\zeta) d \zeta \\
& =\frac{(\mu+1)^{(1-\alpha)+(1-\beta)}}{\Gamma(\alpha) \Gamma(\beta)} \int_{0}^{z}\left(z^{\mu+1}-\zeta^{\mu+1}\right)^{\alpha-1} \zeta^{\mu}\left(\int_{\xi}^{\zeta}\left(\zeta^{\mu+1}-\xi^{\mu+1}\right)^{\beta-1} \xi^{\mu} f(\xi)\right) d \xi d \zeta \\
& =\frac{(\mu+1)^{(1-\alpha)+(1-\beta)}}{\Gamma(\alpha) \Gamma(\beta)} \int_{0}^{z} \xi^{\mu} f(\xi)\left(\int_{\xi}^{\zeta}\left(z^{\mu+1}-\zeta^{\mu+1}\right)^{\alpha-1}\left(z^{\mu+1}-\zeta^{\mu+1}\right)^{\beta-1} \zeta^{\mu}\right) d \zeta d \xi
\end{aligned}
$$

Let $w:=\frac{\zeta^{\mu+1}-\xi^{\mu+1}}{z^{\mu+1}-\xi^{\mu+1}}$, we pose

$$
\begin{aligned}
\int_{\xi}^{\zeta}\left(z^{\mu+1}-\zeta^{\mu+1}\right)^{\alpha-1}\left(\zeta^{\mu+1}-\xi^{\mu+1}\right)^{\beta-1} \zeta^{\mu} d \zeta & =\frac{\left(z^{\mu+1}-\xi^{\mu+1}\right)^{\alpha+\beta-1}}{\mu+1} \int_{0}^{1}(1-\omega)^{\alpha-1} \omega^{\beta-1} d \omega \\
& =\frac{\left(z^{\mu+1}-\xi^{\mu+1}\right)^{\alpha+\beta-1}}{\mu+1} \frac{\Gamma(\alpha) \Gamma(\beta)}{\Gamma(\alpha+\beta)}
\end{aligned}
$$

By (4) and (5), we obtain

$$
\begin{aligned}
I_{z}^{\alpha, \mu} I_{z}^{\beta^{, \mu}} f(z) & =\frac{(\mu+1)^{(2-\alpha-\beta)}}{\Gamma(\alpha) \Gamma(\beta)} \int_{0}^{z} \frac{\left(z^{\mu+1}-\zeta^{\mu+1}\right)^{\alpha+\beta-1}}{\mu+1} \frac{\Gamma(\alpha) \Gamma(\beta)}{\Gamma(\alpha+\beta)} \xi^{\mu} f(\xi) d \xi \\
& =\frac{(\mu+1)^{(2-\alpha-\beta)}}{\Gamma(\alpha) \Gamma(\beta)} \int_{0}^{z} \frac{\left(z^{\mu+1}-\xi^{\mu+1}\right)^{\alpha+\beta-1}}{\mu+1} \frac{\Gamma(\alpha) \Gamma(\beta)}{\Gamma(\alpha+\beta)} \xi^{\mu} f(\xi) d \xi \\
& =\frac{(\mu+1)^{(1-\alpha-\beta)}}{\Gamma(\alpha+\beta)} \int_{0}^{z}\left(z^{\mu+1}-\xi^{\mu+1}\right)^{\alpha+\beta-1} \xi^{\mu} f(\xi) d \xi \\
& =I_{z}^{\alpha+\beta, \mu} .
\end{aligned}
$$


Example 2.1. We find the generalized integral of the function $f(z)=z^{v}, v \in \mathbb{R}$. Let $\eta:=\left(\frac{\zeta}{z}\right)^{\mu+1}$ then

$$
\begin{aligned}
I_{z}^{\alpha, \mu} z^{\nu} & =\frac{(\mu+1)}{\Gamma(\alpha)} \int_{0}^{z}\left(z^{\mu+1}-\zeta^{\mu+1}\right)^{\alpha-1} \zeta^{\mu+v} d \zeta \\
& =\frac{z^{\alpha(\mu+1)+v}}{(\mu+1)^{\alpha} \Gamma(\alpha)} \int_{0}^{1} \frac{v}{\eta+1}(1-\eta)^{\alpha-1} d \eta \\
& =\frac{z^{\alpha(\mu+1)+v}}{(\mu+1)^{\alpha} \Gamma(\alpha)} \int_{0}^{1} \frac{v+\mu+1}{\mu+1}(1-\eta)^{\alpha-1} d \eta \\
& =\frac{z^{\alpha(\mu+1)+v}}{(\mu+1)^{\alpha} \Gamma(\alpha)} B\left(\frac{v+\mu+1}{\mu+1}, \alpha\right) \\
& =\frac{z^{\alpha(\mu+1)+v}}{(\mu+1)^{\alpha} \Gamma(\alpha)} \frac{\Gamma\left(\frac{v+\mu+1}{\mu+1}\right) \Gamma(\alpha)}{\Gamma\left(\alpha+\frac{v+\mu+1}{\mu+1}\right)} \\
& =\frac{z^{\alpha(\mu+1)+v}}{(\mu+1)^{\alpha}} \frac{\Gamma\left(\frac{v+\mu+1}{\mu+1}\right)}{\Gamma\left(\alpha+\frac{v+\mu+1}{\mu+1}\right)},
\end{aligned}
$$

where $B$ is the Beta function. When $\mu=0$, we obtain $I_{z}^{\alpha} z^{v}=z^{\alpha+v} \frac{\Gamma(v+1)}{\Gamma(\alpha+v+1)}$ (see Remark 1.1).

In the next section, we will define generalized fractional derivatives for an arbitrary order. Some of its properties are discussed. Furthermore, applications involving this operator are illustrated.

\section{Generalized differential operator}

Corresponding to the generalized fractional integrals (2), we define the generalized differential operator.

Definition 3.1. The generalized fractional derivative of order $\alpha$ is defined, for a function $f(z)$, by

$$
D_{z}^{\alpha, \mu} f(z):=\frac{(\mu+1)^{\alpha}}{\Gamma(1-\alpha)} \frac{d}{d z} \int_{0}^{z} \frac{\zeta^{\mu} f(\zeta)}{\left(z^{\mu+1}-\zeta^{\mu+1}\right)^{\alpha}} d \zeta ; \quad 0 \leq \alpha<1,
$$

where the function $f(z)$ is analytic in simply-connected region of the complex z-plane C containing the origin, and the multiplicity of $\left(z^{\mu+1}-\zeta^{\mu+1}\right)^{-\alpha}$ is removed by requiring $\log \left(z^{\mu+1}-\zeta^{\mu+1}\right)$ to be real when $\left(z^{\mu+1}-\zeta^{\mu+1}\right)>0$.

Example 3.1. We find the generalized derivative of the function $f(z)=z^{v}, v\lfloor\mathbf{R}$. In the same manner of Example 2.1, we let $\eta:=\left(\frac{\zeta}{z}\right)^{\mu+1}$ then we have 


$$
\begin{aligned}
D_{z}^{\alpha, \mu} z^{v} & =\frac{(\mu+1)^{\alpha}}{\Gamma(1-\alpha)} \frac{d}{d z} \int_{0}^{z} \frac{\zeta^{\mu+\nu}}{\left(z^{\mu+1}-\zeta^{\mu+1}\right)^{\alpha}} d \zeta \\
& =\frac{(\mu+1)^{\alpha-1}}{\Gamma(1-\alpha)} \frac{d}{d z} z^{(1-\alpha)(\mu+1)+\nu} \int_{0}^{1} \frac{\nu+\mu+1}{\mu+1}(1-\eta)^{(1-\alpha)-1} d \eta \\
& =\frac{(\mu+1)^{\alpha-1} \Gamma\left(\frac{v}{\mu+1}+1\right)}{\Gamma\left(\frac{v}{\mu+1}+1-\alpha\right)} z^{(1-\alpha)(\mu+1)+\nu-1} .
\end{aligned}
$$

When $\mu=0$, we obtain $D_{z}^{\alpha} z^{v}=z^{\nu-\alpha} \frac{\Gamma(v+1)}{\Gamma(v+1-\alpha)}$ (see Remark 1.1).

Next, we proceed to prove some relations of the generalized operators $I_{z}^{\alpha, \mu}$ and $D_{z}^{\alpha, \mu}$ for analytic functions of the form

$$
f(z)=\sum_{n=1}^{\infty} a_{n} z^{n}, \quad z \in U .
$$

By employing Theorem 2.2 and Example 2.1, we have the following proposition:

Proposition 3.1. Let $f$ be analytic in $U$ of the form (8). Then,

$$
D_{z}^{\alpha, \mu} I_{z}^{\alpha, \mu} f(z)=I_{z}^{\alpha, \mu} D_{z}^{\alpha, \mu} f(z)=f(z), \quad z \in U .
$$

\section{Applications}

In this section, we discuss some applications of the generalized operators (2) and (7) in geometric function theory and fractional differential equations.

\subsection{Distortion inequalities involving fractional derivatives}

Let $\mathcal{A}$ denote the class of functions $f(z)$ normalized by

$$
f(z)=z+\sum_{n=2}^{\infty} a_{n} z^{n}, \quad z \in U .
$$

Also, let $\mathcal{S}$ and $\mathcal{K}$ denote the subclasses of $\mathcal{A}$ consisting of functions which are, respectively, univalent and convex in $U$. It is well known that if the function $f(z)$ given by (9) is in the class $\mathcal{S}$, then

$$
\left|a_{n}\right| \leq n, \quad n \in \mathbf{N} \backslash\{1\} .
$$

Equality holds for the Koebe function

$$
f(z)=\frac{z}{(1-z)^{2}}, \quad z \in U .
$$

Moreover, if the function $f(z)$ given by (9) is in the class $\mathcal{K}$, then

$$
\left|a_{n}\right| \leq 1, \quad n \in \mathbf{N} .
$$

Equality holds for the function

$$
f(z)=\frac{z}{1-z}, \quad z \in U
$$


In our present investigation, we shall also make use of the Fox-Wright generalization ${ }_{q} \Psi_{p}[z]$ of the hyperge-ometric ${ }_{q} F_{p}$ function defined by [6]

$$
\begin{aligned}
{ }_{q} \psi_{p}\left[\begin{array}{c}
\left(\alpha_{1}, A_{1}\right), \ldots,\left(\alpha_{q}, A_{q}\right) ; \\
\left(\beta_{1}, B_{1}\right), \ldots,\left(\beta_{p}, B_{p}\right) ;
\end{array}\right] & ={ }_{q} \psi_{p}\left[\left(\alpha_{j}, A_{j}\right)_{1, q ;}\left(\beta_{j}, B_{j}\right)_{1, p ; z}\right] \\
: & =\sum_{n=0}^{\infty} \frac{\Gamma\left(\alpha_{1}+n A_{1}\right) \ldots \Gamma\left(\alpha_{q}+n A_{q}\right) z^{n}}{\Gamma\left(\beta_{1}+n B_{1}\right) \ldots \Gamma\left(\beta_{q}+n B_{q}\right) n !} \\
& =\sum_{n=0}^{\infty} \frac{\Pi_{j=1}^{q} \Gamma\left(\alpha_{j}+n A_{j}\right) z^{n}}{\Pi_{j=1}^{p} \Gamma\left(\beta_{j}+n B_{j}\right) n !}
\end{aligned}
$$

where $A_{j}>0$ for all $j=1, \ldots, q, B_{j}>0$ for all $j=1, \ldots, p$ and $1+\sum_{j=1}^{p} B_{j}-\sum_{j=1}^{q} A_{j} \geq 0$ for suitable values $|z|<1$.

Theorem 4.1. Let $f \in \mathcal{S}$. Then,

$$
\begin{gathered}
\left|D_{z}^{\alpha, \mu} f(z)\right| \leq r^{(1-\alpha)(\mu+1)}(\mu+1)^{\alpha-1}\left[\begin{array}{l}
(2,1),\left(1+\frac{1}{\mu+1}, \frac{1}{\mu+1}\right) ; \\
\left(1-\alpha+\frac{1}{\mu+1}, \frac{1}{\mu+1}\right) ;
\end{array}\right], \\
(r=|z| ; z \in U ; 0<\alpha<1),
\end{gathered}
$$

where the equality holds true for the Koebe function.

Proof. Suppose that the function $f(z) \in \mathcal{S}$ is given by (9). Then, by using Example 3.1, we obtain

$$
\begin{aligned}
D_{z}^{\alpha, \mu} f(z) & =\sum_{n=1}^{\infty} \frac{(\mu+1)^{\alpha-1} \Gamma\left(\frac{n}{\mu+1}+1\right)}{\Gamma\left(\frac{n}{\mu+1}+1-\alpha\right)} a_{n} z^{(1-\alpha)(\mu+1)+n-1}, \quad a_{1}=1 \\
& =z^{(1-\alpha)(\mu+1)}(\mu+1)^{\alpha-1} \sum_{n=0}^{\infty} \frac{\Gamma\left(\frac{n+1}{\mu+1}+1\right)}{\Gamma\left(\frac{n+1}{\mu+1}+1-\alpha\right)} a_{n+1} z^{n} .
\end{aligned}
$$

Thus,

$$
\begin{aligned}
\left|D_{z}^{\alpha, \mu} f(z)\right| \leq r^{(1-\alpha)(\mu+1)}(\mu+1)^{\alpha-1} \sum_{n=0}^{\infty} \frac{\Gamma\left(\frac{n+1}{\mu+1}+1\right)}{\Gamma\left(\frac{n+1}{\mu+1}+1-\alpha\right)}(n+1) r^{n} \\
=r^{(1-\alpha)(\mu+1)}(\mu+1)^{\alpha-1} \sum_{n=0}^{\infty} \frac{\Gamma(2+n) \Gamma\left(1+\frac{1}{\mu+1}+\frac{n}{\mu+1}\right) r^{n}}{\Gamma\left(1-\alpha+\frac{1}{\mu+1}+\frac{r^{n}}{\mu+1}\right)} n \\
=r^{(1-\alpha)(\mu+1)}(\mu+1)^{\alpha-1}\left[\begin{array}{l}
(2,1),\left(1+\frac{1}{\mu+1}, \frac{1}{\mu+1}\right) ; \\
\left(1-\alpha+\frac{1}{\mu+1}, \frac{1}{\mu+1}\right) ;
\end{array}\right] \\
=r^{(1-\alpha)(\mu+1)}(\mu+1)^{\alpha-1} 2 \Phi_{1}[r] .
\end{aligned}
$$


In the same manner of Theorem 4.1, we have a distortion inequality involving the Fox-Wright function, which is given by the following:

Theorem 4.2. Let $f \in \mathcal{S}$. Then,

$$
\begin{gathered}
\left|D_{z}^{\alpha+1, \mu} f(z)\right| \leq \frac{(\mu+1)^{\alpha}}{r^{\alpha(\mu+1)}}\left[\begin{array}{l}
(2,1),\left(1+\frac{1}{\mu+1}, \frac{1}{\mu+1}\right) ; \\
\left(\frac{1}{\mu+1}-\alpha \frac{1}{\mu+1}\right) ;
\end{array}\right], \\
(r=|z| ; z \in U ; 0<\alpha<1),
\end{gathered}
$$

where the equality holds true for the Koebe function.

Theorem 4.3. Let $f \in \mathcal{K}$. Then,

$$
\begin{aligned}
& \left|D_{z}^{\alpha+1, \mu} f(z)\right| \leq r^{(1-\alpha)(\mu+1)}(\mu+1)^{\alpha-1}\left[\begin{array}{l}
(1,1),\left(1+\frac{1}{\mu+1}, \frac{1}{\mu+1}\right) ; \\
\left(1-\alpha \frac{1}{\mu+1}-\alpha \frac{1}{\mu+1}\right) ;
\end{array}\right], \\
& (r=|z| ; z \in U ; 0<\alpha<1) \text {, }
\end{aligned}
$$

where the equality holds true for the Koebe function.

Proof. Suppose that the function $f(z) \in \mathcal{K}$. is given by (9). Then, we pose

$$
\begin{aligned}
\left|D_{z}^{\alpha, \mu} f(z)\right| \leq r^{(1-\alpha)(\mu+1)}(\mu+1)^{\alpha-1} \sum_{n=0}^{\infty} \frac{\Gamma\left(\frac{n+1}{\mu+1}+1\right)}{\Gamma\left(\frac{n+1}{\mu+1}+1-\alpha\right)} r^{n} \\
=r^{(1-\alpha)(\mu+1)}(\mu+1)^{\alpha-1} \sum_{n=0}^{\infty} \frac{\Gamma(1+n) \Gamma\left(1+\frac{1}{\mu+1}+\frac{n}{\mu+1}\right) r^{n}}{\Gamma\left(1-\alpha+\frac{1}{\mu+1}+\frac{r^{n}}{\mu+1}\right)} n ! \\
=r^{(1-\alpha)(\mu+1)}(\mu+1)^{\alpha-1}\left[\begin{array}{l}
(1,1),\left(1+\frac{1}{\mu+1}, \frac{1}{\mu+1}\right) ; \\
\left(1-\alpha+\frac{1}{\mu+1}, \frac{1}{\mu+1}\right) ;
\end{array}\right] .
\end{aligned}
$$

\subsection{Fractional differential equations}

In this section, we focus our attention on the fractional differential equation of the form

$$
D_{z}^{\alpha, \mu} u(z)=f(z, u(z))
$$

subject to the initial condition $u(0)=0$, where $u: U \rightarrow \mathbf{C}$ is an analytic function for all $z \in U$, and $f: U \times \mathbf{C} \rightarrow \mathbf{C}$ is an analytic function in $z \in U$. Let $\mathcal{B}$ represent complex Banach space of analytic functions in the unit disk.

Theorem 4.4. (Existence) Let the function $f: U \times \mathbf{C} \rightarrow \mathbf{C}$ be analytic such that $\|f\|$ $\leq M ; M \geq 0$. Then, there exists a function $u: U \rightarrow \mathbf{C}$ solving the problem (15). 
Proof. Define the set $S:\{u \in \mathcal{B}:\|u\| \leq r, r>0\}$, and the operator $P: S \rightarrow S$ by

$$
(P u)(z):=\frac{(\mu+1)^{1-\alpha}}{\Gamma(\alpha)} \int_{0}^{z}\left(z^{\mu+1}-\zeta^{\mu+1}\right)^{\alpha-1} \zeta^{\mu} f(\zeta, u(\zeta)) d \zeta ; \quad \alpha \in(0,1)
$$

First, we show that $P$ is bounded operator:

$$
\begin{aligned}
|(P u)(z)| & =\left|\frac{(\mu+1)^{1-\alpha}}{\Gamma(\alpha)} \int_{0}^{z}\left(z^{\mu+1}-\zeta^{\mu+1}\right)^{\alpha-1} \zeta^{\mu} f(\zeta, u(\zeta)) d \zeta\right| \\
& \leq \frac{M(\mu+1)^{1-\alpha}}{\Gamma(\alpha)} \int_{0}^{z}\left|\left(z^{\mu+1}-\zeta^{\mu+1}\right)^{\alpha-1} \zeta^{\mu}\right| d \zeta \\
& \leq \frac{M(\mu+1)^{1-\alpha}}{\Gamma(\alpha)} B(1, \alpha) \\
& :=r
\end{aligned}
$$

that is $\left\|P_{u}\right\|_{\mathcal{B}}=\sup _{z \in U}|(P u)(z)|$. We proceed to prove that $P: S \rightarrow S$ is continuous operator. Since $f$ is continuous function on $U \times S$, then it is uniformly continuous on a compact set $\tilde{U} \times S$, where

$$
\tilde{U}:=\{z \in U:|z| \leq \ell, 0<\ell<1\} .
$$

Hence, given $\epsilon>0, \exists \delta>0$ such that for all $u, v \in S$ we have

$$
\begin{aligned}
\|f(z, u)-f(z, v)\| & <\frac{\varepsilon \Gamma(\alpha)}{(\mu+1)^{1-\alpha} B(1, \alpha) \ell^{\alpha(\mu+1)}} \quad \text { for }\|u-v\|<\delta, \quad \text { then } \\
|(P u)(z)-(P v)(z)| & =\mid \frac{(\mu+1)^{1-\alpha}}{\Gamma(\alpha)} \int_{0}^{z}\left(z^{\mu+1}-\zeta^{\mu+1}\right)^{\alpha-1} \zeta^{\mu} f(\zeta, u(\zeta)) d \zeta \\
& -\frac{(\mu+1)^{1-\alpha}}{\Gamma(\alpha)} \int_{0}^{z}\left(z^{\mu+1}-\zeta^{\mu+1}\right)^{\alpha-1} \zeta^{\mu} f(\zeta, v(\zeta)) d \zeta \mid \\
& \leq \frac{(\mu+1)^{1-\alpha}}{\Gamma(\alpha)} \int_{0}^{z}\left|\left(z^{\mu+1}-\zeta^{\mu+1}\right)^{\alpha-1} \zeta^{\mu}\right| \times|f(\zeta, u(\zeta))-f(\zeta, v(\zeta))| d \zeta \\
& \leq \frac{\ell^{\alpha(\mu+1)}(\mu+1)^{1-\alpha} B(1, \alpha)}{\Gamma(\alpha)} \times \frac{\varepsilon \Gamma(\alpha)}{(\mu+1)^{1-\alpha} B(1, \alpha) \ell^{\alpha(\mu+1)}} \\
& =\varepsilon .
\end{aligned}
$$

Thus, $P$ is a continuous mapping on $S$. Now, we show that $P$ is an equicontinuous mapping on $S$. For $z_{1}, z_{2} \in \tilde{U}$ such that $z_{1} \neq z_{2}$, then for all $u \in S$ we obtain

$$
\begin{aligned}
\left|(P u)\left(z_{1}\right)-(P u)\left(z_{2}\right)\right| & =\mid \frac{(\mu+1)^{1-\alpha}}{\Gamma(\alpha)} \int_{0}^{z_{1}}\left(z_{1}^{\mu+1}-\zeta^{\mu+1}\right)^{\alpha-1} \zeta^{\mu} f(\zeta, u(\zeta)) d \zeta \\
& -\frac{(\mu+1)^{1-\alpha}}{\Gamma(\alpha)} \int_{0}^{z_{2}}\left(z_{2}^{\mu+1}-\zeta^{\mu+1}\right)^{\alpha-1} \zeta^{\mu} f(\zeta, u(\zeta)) d \zeta \mid \\
& \leq \frac{(\mu+1)^{1-\alpha}}{\Gamma(\alpha)} \int_{0}^{z_{1}}\left|\left(z_{1}^{\mu+1}-\zeta^{\mu+1}\right)^{\alpha-1} \zeta^{\mu} f(\zeta, u(\zeta))\right| d \zeta \\
& +\frac{(\mu+1)^{1-\alpha}}{\Gamma(\alpha)} \int_{0}^{z_{2}}\left|\left(z_{2}^{\mu+1}-\zeta^{\mu+1}\right)^{\alpha-1} \zeta^{\mu} f(\zeta, u(\zeta))\right| d \zeta \\
& \leq \frac{M(\mu+1)^{1-\alpha}}{\Gamma(\alpha)}\left(\int_{0}^{z_{1}}\left|\left(z_{1}^{\mu+1}-\zeta^{\mu+1}\right)^{\alpha-1} \zeta^{\mu}\right| d \zeta+\int_{0}^{z_{2}}\left|\left(z_{2}^{\mu+1}-\zeta^{\mu+1}\right)^{\alpha-1} \zeta^{\mu}\right| d \zeta\right) \\
& \leq \frac{2 M \ell^{\alpha(\mu+1)}(\mu+1)^{1-\alpha}}{\Gamma(\alpha)} B(1, \alpha),
\end{aligned}
$$

which is independent on $u$. Hence, $P$ is an equicontinuous mapping on $S$. The Arzela-Ascoli theorem yields that every sequence of functions from $P(S)$ has got a 
uniformly convergent subsequence, and therefore $P(S)$ is relatively compact. Schauder's fixed point theorem asserts that $P$ has a fixed point. By construction, a fixed point of $P$ is a solution of the initial value problem (15).

Theorem 4.5. (Uniqueness) Let the function $f$ be bounded and fulfill a Lipschitz condition with respect to the second variable: i.e.,

$$
\|f(z, u)-f(z, v)\| \leq L\|u-v\|
$$

for some $L>0$ independent of $u, v$ and $z$. If $\frac{L(\mu+1)^{1-\alpha} B(1, \alpha)}{\Gamma(\alpha)}<1$, then there exists a unique function $u: U \rightarrow \mathbf{C}$ solving the initial value problem (15).

Proof. We need only to prove that the operator $P$ in Equation 3 has a unique fixed point.

$$
\begin{aligned}
|(P u)(z)-(P v)(z)| & =\mid \frac{(\mu+1)^{1-\alpha}}{\Gamma(\alpha)} \int_{0}^{z}\left(z^{\mu+1}-\zeta^{\mu+1}\right)^{\alpha-1} \zeta^{\mu} f(\zeta, u(\zeta)) d \zeta \\
& -\frac{(\mu+1)^{1-\alpha}}{\Gamma(\alpha)} \int_{0}^{z}\left(z^{\mu+1}-\zeta^{\mu+1}\right)^{\alpha-1} \zeta^{\mu} f(\zeta, v(\zeta)) d \zeta \mid \\
& \leq \frac{(\mu+1)^{1-\alpha}}{\Gamma(\alpha)} \int_{0}^{z}\left|\left(z^{\mu+1}-\zeta^{\mu+1}\right)^{\alpha-1} \zeta^{\mu}\right| \times|f(\zeta, u(\zeta))-f(\zeta, v(\zeta))| d \zeta \\
& \leq \frac{L(\mu+1)^{1-\alpha} B(1, \alpha)}{\Gamma(\alpha)}\|u-v\| .
\end{aligned}
$$

Then, for all $u, v$, we obtain

$$
\|P u-P v\| \leq \frac{L(\mu+1)^{1-\alpha} B(1, \alpha)}{\Gamma(\alpha)}\|u-v\| .
$$

Thus, the operator $P$ is a contraction mapping then in view of Banach fixed point theorem, $P$ has a unique fixed point which corresponds to the solution of the initial value problem (15).

\section{Conclusion}

From above, we made a generalization to one of the most important differential and integral operators (Srivastava-Owa operators) of arbitrary order in the unit disk. We found that the generalize integral operator satisfying the semi-group property. Furthermore, their applications appeared in the theory of geometric functions and fractional differential equations by establishing the sufficient conditions for the existence and uniqueness of Cauchy problem in the unit disk.

\section{Acknowledgements}

The author thankful to the anonymous referee for his/her helpful suggestions for the improvement of this article.

\section{Competing interests}

The authors declare that they have no competing interests.

Received: 31 July 2011 Accepted: 15 November 2011 Published: 15 November 2011

\section{References}

1. Darus, M, Ibrahim, RW: Radius estimates of a subclass of univalent functions. Math Vesnik. 63(1):55 (2011)

2. Srivastava, HM, Ling, Y, Bao, G: Some distortion inequalities associated with the fractional derivatives of analytic and univalent functions. J Inequal Pure Appl Math. 2(2):1 (2001)

3. Srivastava, HM, Owa, S: Univalent Functions, Fractional Calculus, and Their Applications. Halsted Press, Wiley, New York (1989) 
4. Ibrahim, RW, Darus, M: Subordination and superordination for analytic functions involving fractional integral operator. Complex Variables Elliptic Equations. 53(11):1021 (2008). doi:10.1080/17476930802429131

5. Ibrahim, RW, Darus, M: Subordination and superordination for univalent solutions for fractional differential equations. J Math Anal Appl. 345(2):871 (2008). doi:10.1016/j.jmaa.2008.05.017

6. Srivastava, HM, Karlsson, PW: Multiple Gaussian Hypergeometric Series. Halsted Press, Ellis Horwood Limited, Chichester, Wiley, New York (1985)

doi:10.1186/1687-1847-2011-55

Cite this article as: Ibrahim: On generalized Srivastava-Owa fractional operators in the unit disk. Advances in Difference Equations 2011 2011:55.

Submit your manuscript to a SpringerOpen ${ }^{\circ}$ journal and benefit from:

- Convenient online submission

- Rigorous peer review

- Immediate publication on acceptance

- Open access: articles freely available online

- High visibility within the field

- Retaining the copyright to your article

Submit your next manuscript at $\gg$ springeropen.com 